\title{
Hidden Hungers of Camden: A Pilot Intervention unraveling the double burden of Malnutrition Among Ethnic Minority Women living in Deprivation
}

\author{
C. Browne and I. Twefik \\ School of Life Sciences; Division of Food, Nutrition, and Public Health University of Westminster, London W1 W \\ $6 U W$
}

Women are generally more at risk for nutritional deficiencies and poor health due to gender bias and systematic discrimination; placing them at a lower worth in society than $\operatorname{men}^{(1)}$. Using a mixed methods approach, (quantitative and qualitative) this study explores the phenomenon of demographics and socio-economic status and its impact on nutrient intake among ethnic minority women. This intervention addresses research gaps that include lack of data of nutritional status for ethnic minority women groups living in the UK and subsequently limited tailored nutrition interventions targeting them.

Forty-two Women were recruited by non-probability sampling approached in person in community centres within deprived wards of Camden, London, UK. Inclusion/Exclusion criteria included; gender (female) aged 25-65 years, living in deprived wards of Camden, unemployed, or employed (low-income earning annual household income between $£ 5,200-£ 10,399$ ).

This pilot was a six-week intervention referred to with the slogan "Woman Nutrition Programme" (WNP) objectives included; increase micro-nutrient intake through weekly nutrition education and healthy cooking sessions tailored towards ethnic sub-groups of the cohort as well as positively influence household nutritional status. The intervention also included physical activities, interactive workshops, and women empowerment sessions.

Quantitive Data was collected using 24-dietary recalls as the main nutritional assessment tool pre and post intervention to measure nutrient intake, data coded using Nutritics, nutritional analysis software. Anthropometric measurements were taken pre and post study including; Body Mass Index (BMI), Waist Circumference (WC), height, and weight. Other quantitative measures included eating habits questionnaires.

Qualitative Data collection included focus group discussions, one to one interviews, and case studies.

Statistical analysis completed, using IBM SPSS software (version 24) at 95\% confidence level p >0.05. Descriptive Statistics were expressed as mean \pm standard deviation.

Based on 24-hour dietary recalls, used as main nutritional assessment tool, average mean nutrient intake were lower range of the normal value of recommended UK reference values for nutrients; calcium, folic acid (vitamin B9), iron, magnesium, potassium, and vitamin D.

Collected data of recruited participants [ $\mathrm{N}=42]$ showed that: At baseline; $23 \%$ were classified as obesity class I [BMI 30.0-34.9 $\left.\mathrm{kg} / \mathrm{m}^{2}\right]$ and $40 \%$ pre-obese [BMI $35.0-39.9 \mathrm{~kg} / \mathrm{m}^{2}$ ]. $21 \%$ had an average WC $>80 \mathrm{~cm}$ while $55 \%$ had WC $>88 \mathrm{~cm}$. $67 \%$ of women were unemployed and $23 \%$ reported Level 3 education qualifications.

Qualitative data collected in the form of focus group discussions and case-studies revealed that based on traditional cooking women were consuming nutrient deficient meals each day. Being overweight was also perceived as "healthy weight" within cohort group which may contribute towards unhealthy eating behaviours.

In conclusion participants that completed $(\mathrm{N}=23)$, WNP, showed a significant increase in nutrient intake for example; potassium mean intake $2073.43 \mathrm{mg}$ (pre) and $2279.43 \mathrm{mg}$ (post). Results from the study reveal a double burden of malnutrition[1]. Therefore, developing culturally tailored nutrition interventions which include skills based healthy cooking courses and nutrition education could positively increase micro-nutrient intake among those suffering from hidden hungers.

1. Darnton-Hill, et al. (2005). Micronutrient deficiencies and gender: social and economic costs. The American Journal of Clinical Nutrition 81(5), 1198S-1205S. Available from 10.1093/ajcn/81.5.1198. 